City University of New York (CUNY) CUNY Academic Works

2012

\title{
A New Body Shape Index Predicts Mortality Hazard Independently of Body Mass Index
}

Nir Y. Krakauer

CUNY City College

Jesse C. Krakauer

Middletown Medical

\section{How does access to this work benefit you? Let us know!}

More information about this work at: https://academicworks.cuny.edu/cc_pubs/39

Discover additional works at: https://academicworks.cuny.edu

This work is made publicly available by the City University of New York (CUNY).

Contact: AcademicWorks@cuny.edu 


\title{
A New Body Shape Index Predicts Mortality Hazard Independently of Body Mass Index
}

\author{
Nir Y. Krakauer ${ }^{1 *}$, Jesse C. Krakauer ${ }^{2}$
}

1 Department of Civil Engineering, The City College of New York, New York, New York, United States of America, 2 Middletown Medical, Middletown, New York, United States of America

\begin{abstract}
Background: Obesity, typically quantified in terms of Body Mass Index (BMI) exceeding threshold values, is considered a leading cause of premature death worldwide. For given body size (BMI), it is recognized that risk is also affected by body shape, particularly as a marker of abdominal fat deposits. Waist circumference (WC) is used as a risk indicator supplementary to BMI, but the high correlation of WC with BMI makes it hard to isolate the added value of WC.
\end{abstract}

Methods and Findings: We considered a USA population sample of 14,105 non-pregnant adults (age $\geq 18$ ) from the National Health and Nutrition Examination Survey (NHANES) 1999-2004 with follow-up for mortality averaging 5 yr (828 deaths). We developed A Body Shape Index (ABSI) based on WC adjusted for height and weight:

$$
\mathrm{ABSI} \equiv \frac{\mathrm{WC}}{\mathrm{BMI}^{2 / 3} \text { height }^{1 / 2}}
$$

ABSI had little correlation with height, weight, or BMI. Death rates increased approximately exponentially with above average baseline ABSI (overall regression coefficient of $+33 \%$ per standard deviation of ABSI [95\% confidence interval: $+20 \%-$ $+48 \%])$, whereas elevated death rates were found for both high and low values of BMI and WC. $22 \%(8 \%-41 \%)$ of the population mortality hazard was attributable to high ABSI, compared to $15 \%(3 \%-30 \%)$ for BMI and $15 \%(4 \%-29 \%)$ for WC. The association of death rate with $A B S I$ held even when adjusted for other known risk factors including smoking, diabetes, blood pressure, and serum cholesterol. ABSI correlation with mortality hazard held across the range of age, sex, and BMI, and for both white and black ethnicities (but not for Mexican ethnicity), and was not weakened by excluding deaths from the first 3 yr of follow-up.

Conclusions: Body shape, as measured by ABSI, appears to be a substantial risk factor for premature mortality in the general population derivable from basic clinical measurements. ABSI expresses the excess risk from high WC in a convenient form that is complementary to $\mathrm{BMI}$ and to other known risk factors.

Citation: Krakauer NY, Krakauer JC (2012) A New Body Shape Index Predicts Mortality Hazard Independently of Body Mass Index. PLoS ONE 7(7): e39504. doi:10.1371/journal.pone.0039504

Editor: Shengxu Li, Tulane School of Public Health and Tropical Medicine, United States of America

Received January 19, 2012; Accepted May 25, 2012; Published July 18, 2012

Copyright: (c) 2012 Krakauer, Krakauer. This is an open-access article distributed under the terms of the Creative Commons Attribution License, which permits unrestricted use, distribution, and reproduction in any medium, provided the original author and source are credited.

Funding: The authors have no funding or support to report.

Competing Interests: JCK is employed by commercial company "Middletown Medical". This does not alter the authors' adherence to all the PLoS ONE policies on sharing data and materials.

*E-mail: nkrakauer@ccny.cuny.edu

\section{Introduction}

According to the World Health Organization (WHO), overweight and obesity are increasing in prevalence and rank fifth as worldwide causes of death among risk factors, behind high blood pressure, tobacco use, high blood glucose, and physical inactivity. In high and middle income countries, where the prevalence of overweight and obesity among the adult population already exceeds $50 \%$, overweight and obesity occupy third place as risk factors causing death, behind high blood pressure and tobacco use. WHO defined overweight as body mass index (BMI; weight divided by height ${ }^{2}$ ) at or above $25 \mathrm{~kg} \mathrm{~m}^{-2}$, with obesity defined as $\mathrm{BMI} \geq 30 \mathrm{~kg} \mathrm{~m}^{-2}$ [1]. Guidelines published by the USA National Institutes of Health, using the same definition, considered that overweight and obesity are the second leading cause of preventable death in the USA, behind smoking [2].

These BMI-based obesity guidelines have been accompanied by doubt as to the validity of BMI as an indicator of dangerous obesity. BMI does not distinguish between muscle and fat accumulation [3-6], and there is evidence that whereas higher fat mass is associated with greater risk of premature death, higher muscle mass reduces risk [7]. As well, BMI does not distinguish between fat locations, when central or abdominal fat deposition is thought to be particularly perilous [8-11]. Waist circumference (WC) has emerged as a leading complement to BMI for indicating obesity risk. A number of studies have found that WC predicted mortality risk better than BMI [12-17]. A recent WHO report summarized evidence for $\mathrm{WC}$ as an indicator of disease risk, and suggests that WC could be used as a alternative to BMI [18]. 
A key limitation, mentioned in the WHO report, of using WC as a proxy for abdominal fat distribution is that it is sensitive to body size (height and weight) as well as to fat percentage and distribution. In fact, WC is highly correlated with BMI, to the extent that differentiating the two as epidemiological risk factors can be difficult [19]. According to a consensus statement on the clinical usefulness of WC [20], "Further studies are needed to establish WC cut points that can assess cardiometabolic risk, not adequately captured by BMI and routine clinical assessments." Scaling WC via allometric analysis to produce a quantity that is independent of BMI offers one means of separating the impact on health of body shape (degree of central bulge, presumably correlating with abdominal fat deposits) from that of body size (as measured by height, weight, and BMI). In this paper, our objectives are (1) develop A Body Shape Index (ABSI) based on WC that is approximately independent of height, weight, and BMI; and (2) evaluate ABSI as a predictor of mortality across age, sex, ethnicity, and BMI categories in a population sample, compared to the conventional predictors BMI and WC.

\section{Methods}

\section{Description of Data}

We employed public-use releases of baseline interview and medical examination and mortality outcome data from the National Health and Nutrition Examination Survey (NHANES) 1999-2004. NHANES 1999-2004 was designed to sample the civilian noninstitutionalized USA population using a cluster approach. Mexicans and blacks, people 12-19 years of age and 60 years or older, low-income whites, and pregnant women were oversampled to better understand the health status of these groups. The survey included a home interview followed by a physical examination at a mobile examination center. Mortality outcomes based on the National Death Index were available through the end of 2006, representing 2-8 years of follow-up. NHANES 19992004 was approved by the National Center for Health Statistics (NCHS) Research Ethics Review Board under Protocol \#98-12, and written informed consent was obtained from participants [21].

Basic demographic variables included baseline age (because of privacy concerns, this was given as 85 for all those 85 or older), sex, and ethnicity (given as Mexican, other Hispanic, white, black, or other). Body measurements including height, weight, and waist circumference were obtained by trained health technicians following standardized procedures. Standing height was measured using a digital stadiometer with a fixed vertical backboard and an adjustable head piece. Weight in an examination gown was measured on a digital scale. Waist circumference at the end of a normal exhalation was measured to the nearest $0.1 \mathrm{~cm}$ with a steel tape positioned just above the uppermost lateral border of the ilium. All instruments were calibrated following uniform protocols. These body measurements were generally not taken for people confined to wheelchairs [22].

We considered adults (age $\geq 18$ ) with height, weight, and waist circumference measurements, excluding women determined to be pregnant by self-report or urine pregnancy test. Of the 14,123 individuals meeting these criteria, 14,105 had valid mortality follow-up data. The follow-up period for those remaining alive averaged $4.8 \mathrm{yr}$, and there were 828 deaths.

Additional variables we considered were smoking status (coded as yes if reported smoking cigarettes in last 5 days or smoking 'every day' or 'some days'), diabetes status (coded yes if reported a diabetes diagnosis or taking insulin or diabetes pills), and systolic and diastolic blood pressure and serum total and HDL cholesterol from the medical examination. A total of 12,044 individuals in our sample had all these variables available, with 647 deaths during follow-up.

We used the NHANES mobile examination center sample weights, which adjust for targeted oversampling and nonresponse, as well as information on which cluster each sampled individual belonged to, following the analytic guidance provided by NCHS [23]. Thus, the average ABSI values and risk estimates we compute can be taken to hold for the wider nonpregnant adult USA population insofar as NHANES was successful in sampling it. Looking at the consistency of responses across subgroups within the sample can also provide some guidance on the wider applicability of our results.

\section{Construction of the Body Shape Index}

We performed linear least-squares regression on $\log (\mathrm{WC})$ as a function of $\log$ (height) and $\log$ (weight) for the entire nonpregnant adult sample. (Pregnant woman averaged bigger WC for a given height and weight.) Expressing $\mathrm{WC}$ and height in $\mathrm{m}$ and weight in $\mathrm{kg}$, the results were.

$$
\begin{aligned}
\log (\mathrm{WC}) & =(-2.589 \pm 0.020)+(0.6807 \pm 0.0052) \log (\text { weight }) \\
& -(0.814 \pm 0.020) \log (\text { height })
\end{aligned}
$$

$\left(R^{2}=0.829\right)$, where the given uncertainties are standard errors. Approximating the obtained regression coefficients with ratios of small integers, we have,

$$
\text { WC } \propto \text { weight } t^{2 / 3} \text { height }^{-5 / 6} \text {. }
$$

We defined A Body Shape Index (ABSI) to be proportional to the ratio of actual $\mathrm{WC}$ to the $\mathrm{WC}$ expected from the regression allometry:

$$
\mathrm{ABSI} \equiv \mathrm{WC} \text { weight }^{-2 / 3} \text { height }^{5 / 6}=\frac{\mathrm{WC}}{\mathrm{BMI}^{2 / 3} \text { height }^{1 / 2}} .
$$

The sample mean and standard deviation of ABSI thus defined is $(0.0808 \pm 0.0053) \mathrm{m}^{11 / 6} \mathrm{~kg}^{-2 / 3}$.

Correlation coefficients of ABSI with height, weight, BMI and WC in the NHANES sample are shown in Table 1. It can be seen that most variability in WC reflects variability in $\mathrm{BMI}(r \approx 0.9)$ and that unlike BMI, WC also has some correlation with height $(r \approx 0.2)$, consistent with earlier findings [24-26]. On the other

Table 1. Correlations between body size and shape.

\begin{tabular}{llllll}
\hline & Height & Weight & BMI & WC & ABSI \\
\hline Height & 1 & 0.452 & -0.040 & 0.174 & 0.040 \\
Weight & 0.380 & 1 & 0.867 & 0.874 & 0.049 \\
BMI & 0.007 & 0.922 & 1 & 0.881 & 0.019 \\
WC & 0.163 & 0.908 & 0.918 & 1 & 0.439 \\
ABSI & 0.041 & 0.020 & 0.007 & 0.361 & 1 \\
\hline
\end{tabular}

Correlation coefficients between height, weight, BMI, WC, and ABSI among NHANES nonpregnant adults ( $n=14123$ ). Right side (above diagonal) shows correlations of the raw values; left side (below diagonal) shows correlations of the $z$ scores relative to age- and sex-specific means.

doi:10.1371/journal.pone.0039504.t001 
hand, ABSI shows little correlation with height, weight, or BMI $(|r|<0.1)$. Its correlation with WC is modest $(r \approx 0.4)$, since most variability in WC is correlated with $\mathrm{BMI}$ and therefore excluded from ABSI.

\section{Conversion to z Scores}

To control for age and sex differences in mean ABSI, we entered it into proportional hazards regression for mortality as a z score:

$$
\mathrm{ABSI} z \text { score }=\frac{\mathrm{ABSI}-\mathrm{ABSI}_{\text {mean }}}{\mathrm{ABSI}},
$$

where the population ABSI mean and standard deviation depend on age and sex. To estimate $\mathrm{ABSI}_{\text {mean }}$ and $\mathrm{ABSI}_{\mathrm{SD}}$, we first computed the sample mean and standard deviation for each age, separately for males and females and using the NHANES sample weights (markers in Figure la-b). Then we smoothed the $\mathrm{ABSI}_{\text {mean }}$ (age) and $\mathrm{ABSI}_{\mathrm{SD}}^{2}$ (age) curves for each sex using Tikhonov regularization with a regularization matrix that approximates a second derivative operator and a regularization parameter chosen so that the mean square residual between the curve and the sample values, scaled by the estimated standard error of the sample values, is equal to 1 [27]. These smoothed values (curves in Figure 1) were used for converting ABSI to $\mathrm{z}$ scores following Eq. 4. Individuals age 85 and over (for whom the exact age was not available) were not included in the smoothing, and their ABSI values were converted to $\mathrm{z}$ scores using the sample mean and standard deviation (asterisks near right edges of panels in Figure 1). The age and sex specific $\mathrm{ABSI}_{\text {mean }}$ and $\mathrm{ABSI}_{\mathrm{SD}}$ used for computing ABSI z scores are tabulated as (Table $\mathrm{S} 1$ ).

Mean ABSI increased steadily from midlife into old age (Figure 1a). Mean ABSI was consistently higher in males than females after young adulthood (Figure 1a), while the scatter in ABSI at a given age was greater in females than in males (Figure 1b). The age- and sex-specific BMI and WC means (Figure 1c,e), calculated using the same approach, showed different behavior than ABSI, falling after about age 60. Mean WC was higher in males while mean BMI was higher in females, consistent with the higher mean ABSI in males compared to females. As with ABSI, variability in BMI and WC was higher in females than in males (Figure 1d,f).

\section{Mortality Hazard Modeling}

To quantify the association of baseline ABSI with death rate, we employed Cox proportional hazard modeling for mortality with age as the time scale [28]. In this approach, log death rate is modeled as a nonparametric function of age plus fitted coefficients that multiply the values of predictors, such as baseline ABSI. Predictors may be entered as continuous variables or discretized into two or more categories (such as quantiles of ABSI), depending on their nature and the desired model. ABSI and the other anthropometric variables (BMI, WC) were entered as $\mathrm{z}$ scores relative to age- and sex-specific normals, obtained as described above, to avoid confounding by age and sex differences in body size and shape.

Two types of models were employed. In one ('unadjusted'), the anthropometric variable of interest (ABSI, BMI, or WC) was the only predictor entered. In the second ('adjusted'), additional predictors corresponding to other known major risk factors were entered. Comparing the unadjusted and adjusted model coefficients showed to what extent the mortality risk associated with higher ABSI, BMI, or WC changed when these other risk factors are controlled for. The additional factors considered were sex, ethnicity, smoking status, presence of diabetes, blood pressure, and serum cholesterol. Sex, ethnicity, smoking status, and presence of diabetes were all entered as binary variables. Ethnicity was entered as 1 for blacks and 0 for all others, since we found that blacks had significantly elevated death rates compared to the other four ethnicities, whose death rates were not significantly different from each other. Systolic and diastolic blood pressure and total and HDL cholesterol levels were each entered as z scores relative to age- and sex-specific normals, obtained as described for ABSI. Because not all individuals with anthropometric measurements also had the other data needed for the adjusted analysis, the unadjusted analyses were run twice - once for the full sample with available anthropometry $(n=14105)$ and once restricted to the sample used for the adjusted analysis $(n=12044)$.

We also determined the mortality risk associated with ABSI, BMI, and WC for subgroups of the NHANES sample, in order to test the robustness and range of applicability of coefficients determined for the entire sample. Subgroups included males and females; people younger and older than $65 \mathrm{yr}$ at baseline; the three largest ethnic groupings (whites, blacks, and Mexicans); and people with $\mathrm{BMI}$ above and below the age- and sex-specific mean. As another check of whether these attributes impact the association with mortality, we checked the significance in the proportional hazard model of interaction terms of ABSI (BMI, WC) with sex, age, ethnicity variables (white, black, or Mexican), and BMI. To address the question of whether ABSI predicts medium-term as compared to short-term mortality, we conducted an additional analysis where the modeled follow-up period started $3 \mathrm{yr}$ after the baseline, thus excluding from consideration all deaths within $3 \mathrm{yr}$ of examination.

In Cox proportional hazard modeling, the relationship between hazard (here, death rate) and continuous variables, such as ABSI here, is most commonly estimated on the assumption that the logarithm of the hazard is a linear function of the variable; this yields a single regression coefficient that summarizes the strength of the relationship between the variable and log hazard. A recommended test of this linearity assumption is to fit an alternative model where the dependence of log hazard on the variable is described by a smoothing spline, with the degree of smoothing determined to optimize the Akaike Information Criterion [29,30]. Linearity is rejected if the nonlinear terms in the fitted smoothing spline are different from zero with low $\mathrm{p}$ value. Our testing showed that the linearity assumption did not hold for ABSI, BMI or WC. In showing results from the models described above, we retained the linearity assumption for all three variables to facilitate comparing mortality hazards across populations and population subgroups. In separate analyses, we also fit smoothing splines to the association with mortality risk of ABSI, $\mathrm{BMI}$, and WC in order to visualize it as accurately as possible. To quantify in a simpler form the nonlinear relationship between ABSI (BMI, WC) and log mortality, we also carried out analyses where risk was computed separately for each quintile of the ABSI (BMI, WC) z score, relative to the middle quintile.

A measure of the fraction of the total population mortality hazard predicted by high values of ABSI (BMI, WC) was calculated as.

Fraction population mortality hazard from high values

$=\frac{\sum_{i, \mathrm{high}} f_{i}\left(R_{i}-1\right)}{\sum_{i, \mathrm{all}} f_{i} R_{i}}$

where $f_{i}$ is the fraction of the population in each quantile and $R_{i}$ is the relative risk in each quantile. The quantiles we used for this 
(a) ABSI mean

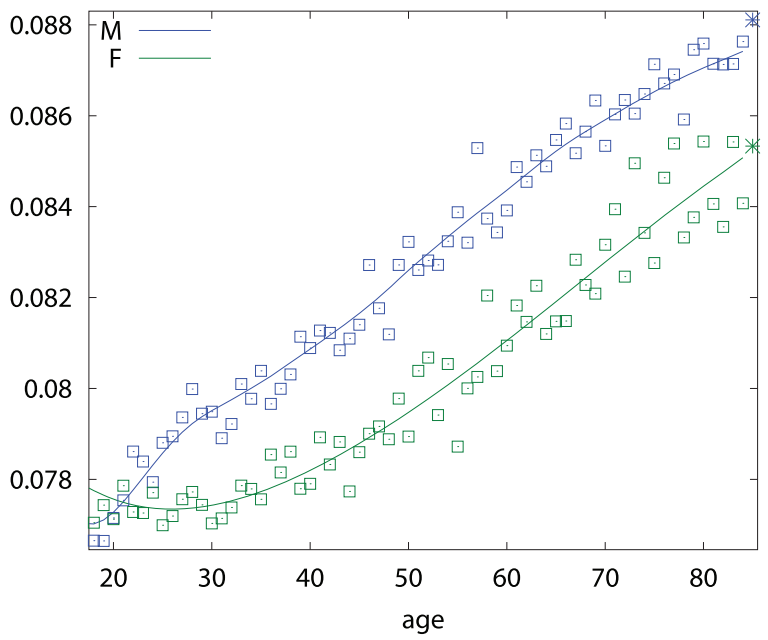

(c) BMI mean

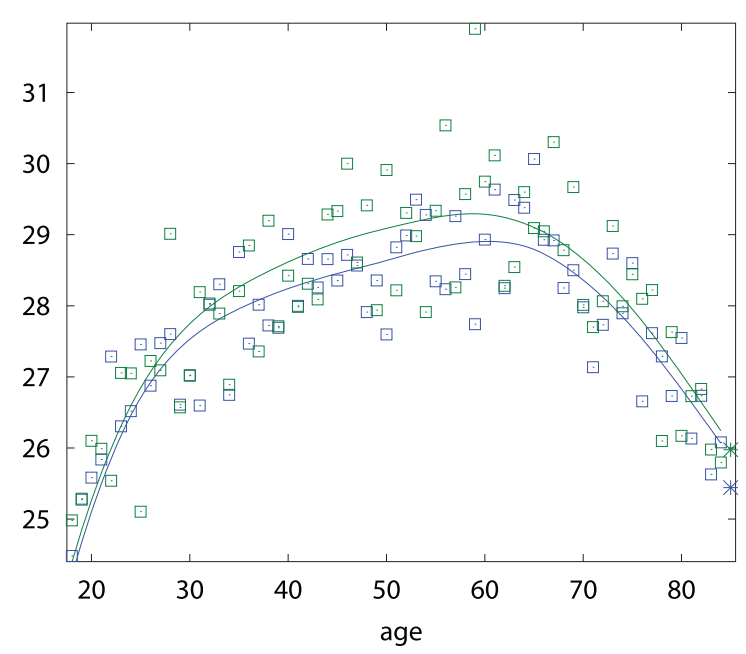

(e) WC mean

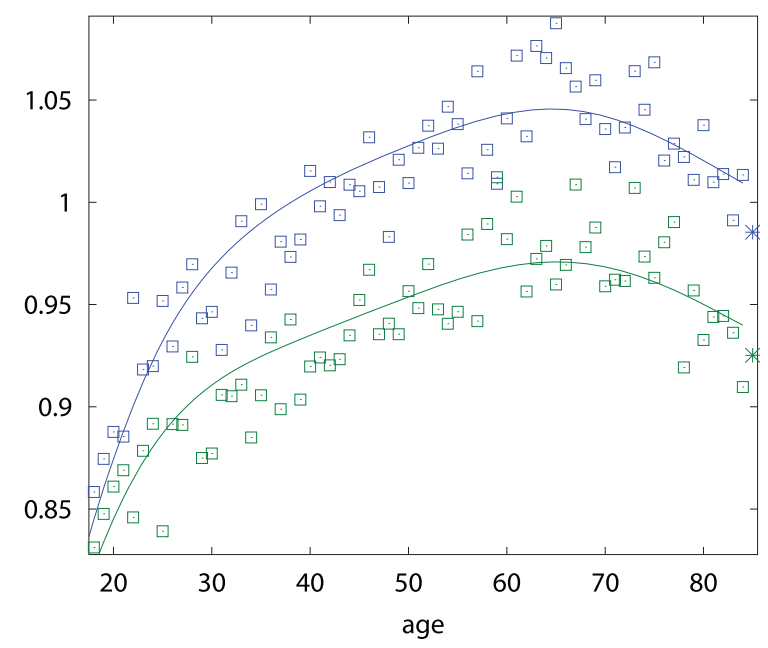

(b) ABSI SD

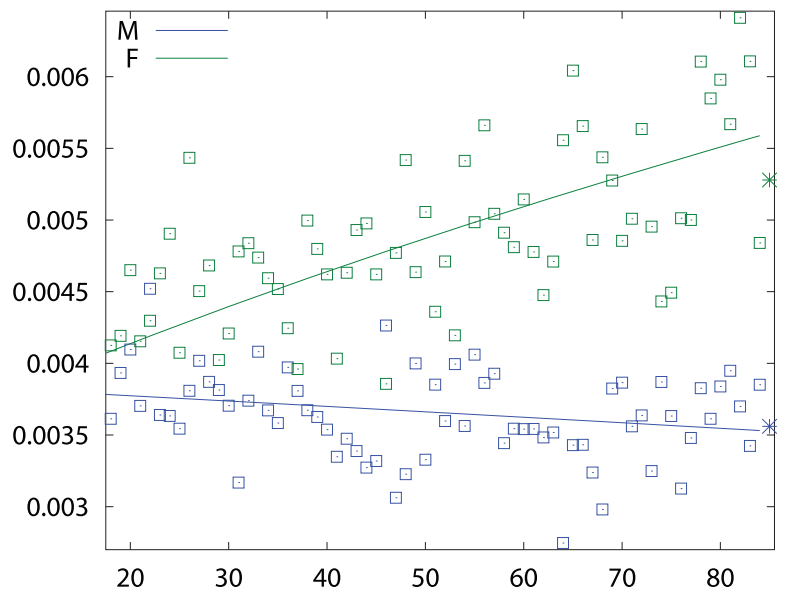

(d) BMI SD

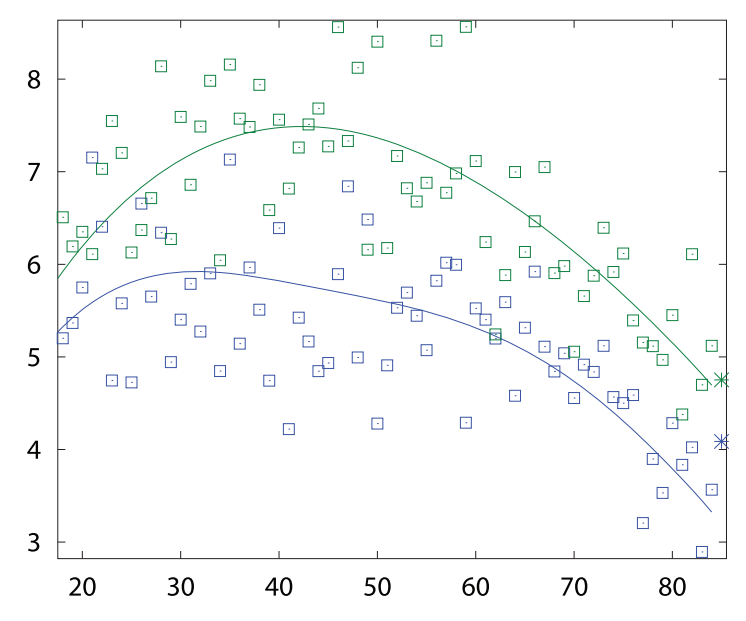

(f) WC SD

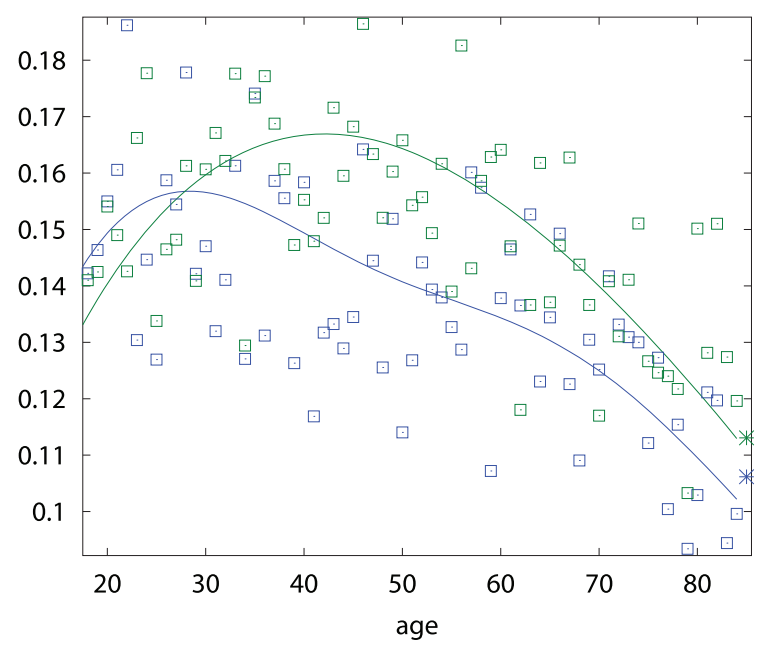

Figure 1. Mean and standard deviation of ABSI, BMI, and WC by age and sex. Markers show the sample quantities for each age; the smooth curves shown were used to convert values to $z$ scores. Units are $\mathrm{m}^{11 / 6} \mathrm{~kg}^{-2 / 3}$ for $A B S l, \mathrm{~kg} \mathrm{~m}^{-2}$ for BMl, and $\mathrm{m}$ for WC. doi:10.1371/journal.pone.0039504.g001 
calculation were the top $40 \%$, middle $20 \%$, and bottom $40 \%$, with the top $40 \%$ regarded as the 'high' range included in the numerator, and risks were relative to the middle quintile. Uncertainty in this expression was approximated as being due only to uncertainty in the numerator $R_{i}$.

While converting variables to z scores before entering them into a hazard regression model may be methodologically preferable given the nonlinear effects of age and sex on mean ABSI, BMI, and WC (Figure 1), we also conducted the same proportional hazard modeling using the original variables, rather than $\mathrm{z}$ scores, as predictors. For these analyses, sex was included as a predictor even for the unadjusted models, in order to control for the sex differences in ABSI, BMI, and WC distributions.

Proportional hazard modeling, including differential sample weighting and adjustment for the cluster survey design, was carried out using the survey package in the computer language R [31]. For all analyses, $p<0.05$ (two-tailed) was taken as the threshold for statistical significance. This corresponds to the $95 \%$ confidence interval of the exponent of a linear regression $\log$ hazard coefficient not including 1 (as would be the case under the null hypothesis that the variable has no effect on the death rate).

\section{Results}

\section{Higher Mortality Hazard for Increasing ABSI}

Table 2 shows the impact of ABSI $\mathrm{z}$ score, as a continuous variable, on death rate, along with results for $\mathrm{BMI}$ and $\mathrm{WC}$ $\mathrm{z}$ scores. ABSI clearly has distinct impacts on mortality compared to BMI and WC: if we model the relationship between the $\mathrm{z}$ scores and $\log$ mortality risk as linear, the regression coefficients imply that uniformly increasing the population ABSI by one standard deviation would result in a significant increase of 33\% $(95 \%$ confidence interval: $+20 \%-+48 \%$ ), while the corresponding linear regression coefficients for BMI and WC are not significantly different from zero. Because the proportional hazard regression coefficients for ABSI, BMI, and WC showed little impact generally shifting by less than their standard error - from either restricting the sample to those with data for the other risk factors (sex, ethnicity, smoking, diabetes, blood pressure, and serum cholesterol; middle column of Table 2) or from adjusting for the other risk factors (right column of Table 2), we carried out the analyses described below with unadjusted models.

\section{Mortality Hazard from Increasing ABSI by Subgroup}

Restricting our analysis to males or females within the sample does not significantly change the impact of increasing ABSI on

Table 2. Body size and shape z scores and mortality hazard.

\begin{tabular}{|c|c|c|c|}
\hline & \multicolumn{3}{|c|}{ Hazard ratio per SD increase } \\
\hline & Unadjusted & Restricted & Adjusted \\
\hline ABSI & $1.33(1.20-1.48)$ & $1.37(1.23-1.53)$ & $1.30(1.16-1.44)$ \\
\hline BMI & $0.98(0.89-1.08)$ & $0.98(0.88-1.09)$ & $0.96(0.86-1.08)$ \\
\hline WC & $1.07(0.98-1.16)$ & $1.08(0.98-1.20)$ & $1.05(0.94-1.17)$ \\
\hline \multicolumn{4}{|c|}{$\begin{array}{l}\text { Results of Cox proportional hazard modeling for mortality risk with ABSI, BMI, or } \\
\text { WC z scores taken as linear predictors. Ranges in parentheses are } 95 \% \\
\text { confidence intervals. The restricted models are unadjusted but included only } \\
\text { those people who had all the measurements required for the adjusted model } \\
\text { The adjusted models included as additional predictors sex, ethnicity, smoking } \\
\text { presence of diabetes, blood pressure, and serum cholesterol. } \\
\text { SD = standard deviation. } \\
\text { doi:10.1371/journal.pone. } 0039504.002\end{array}$} \\
\hline
\end{tabular}

mortality (Table 3); the change in mortality hazard per standard deviation increase in ABSI is $+32 \%$ (95\% confidence interval: $+15 \%-+50 \%)$ for males and $+35 \%$ (95\% confidence interval: $+18 \%-+54 \%$ ) for females. Further, high ABSI predicts similar elevation of relative mortality hazard for younger (age $<65 \mathrm{yr}$ at baseline) and older (age $\geq 65 \mathrm{yr}$ ) individuals, with narrower confidence intervals for the older group because of their much higher absolute death rate over the follow-up period (Table 3). ABSI predicted mortality among individuals with above-mean BMI about as well as it did for individuals with below-mean BMI (Table 3). Among the three main ethnic groups in the sample, ABSI predicted mortality in both whites and blacks, while ABSI was not a significant predictor of mortality in Mexicans (Table 3).

These conclusions from subgroup analysis were largely borne out by checking the significance of interaction terms added to the Cox proportional hazard model. ABSI*age, ABSI*sex, and ABSI $*$ BMI interactions were not significant, confirming that high ABSI predicts mortality across these categories. By contrast, the impact of increasing BMI and WC depended strongly on BMI (Table 3), consistent with U-shaped relationships where lower weight would increase mortality at low BMI and decrease it at high BMI. Interaction with ABSI of an indicator variable for white ethnicity were significantly positive, implying that whites with high ABSI show greater relative risk elevation than other USA ethnicities.

High ABSI continued to be a significant predictor of death even when the first $3 \mathrm{yr}$ of the follow-up period were excluded (Table 3), suggesting that the correlation of higher ABSI with death rate is not merely due to a propensity of acutely ill people to have high ABSI.

\section{Mortality Hazard by ABSI Quantile}

To examine the correlation of different levels of ABSI with death rate, we stratified the population into quintiles by ABSI z score, where the middle (third) quintile included those near the population mean ABSI, and conducted proportional hazard modeling with ABSI quintiles, rather than ABSI z score, as the predictor variables, with hazard ratios expressed relative to the middle quintile. We found that people with low ABSI (first and second quintiles) had nonsignificantly decreased mortality risk relative to the middle quintile, while ABSI in the fourth and fifth quintiles was associated with progressively and significantly increased mortality risk (Table 4).

Similar analyses were conducted with BMI and WC quintiles. $\mathrm{BMI}$ and WC in the first quintile were both associated with significantly greater mortality hazard than for the middle quintile. Significantly increased death rate compared to the middle quintile was also seen for WC and BMI in the fifth quintile (Table 4). Comparing the excess mortality hazard from high ABSI (top two quintiles) with that posed by high $\mathrm{BMI}$ and $\mathrm{WC}$ as a fraction of the total population death rate, we found that $22 \%(8 \%-41 \%)$ of the population mortality hazard was attributable to high ABSI, compared to $15 \%(3 \%-30 \%)$ for BMI and $15 \%(4 \%-29 \%)$ for WC. Plotting estimated mortality risk by percentile from models with ABSI, BMI, or WC as continuous predictor variables (Figure 2), with smoothing splines used to represent their nonlinear associations with mortality, gives results consistent with the quantile analyses. While risk increases progressively with increasing ABSI, BMI and WC risk is lowest near the population median and increases for both high and low values.

Figure 3 shows the estimated relative mortality risk taking both body size (BMI) and shape (ABSI) z score into account. These estimates are based on the lack of interaction we found between BMI and ABSI as predictors of mortality, so that the estimated 
Table 3. Mortality hazard by subgroup.

\begin{tabular}{|c|c|c|c|c|}
\hline & \multicolumn{4}{|c|}{ Hazard ratio } \\
\hline & Deaths/N & ABSI & BMI & wC \\
\hline All & $828 / 14105$ & $1.33(1.20-1.48)$ & $0.98(0.89-1.08)$ & $1.07(0.98-1.16)$ \\
\hline Male & $502 / 7133$ & $1.32(1.15-1.50)$ & $0.92(0.81-1.06)$ & $1.00(0.88-1.14)$ \\
\hline Female & $326 / 6972$ & $1.35(1.18-1.54)$ & $1.04(0.91-1.19)$ & $1.14(1.01-1.30)$ \\
\hline$<65 \mathrm{yr}$ & $213 / 10728$ & $1.37(1.12-1.69)$ & $1.05(0.84-1.31)$ & $1.12(0.91-1.39)$ \\
\hline$\geq 65 \mathrm{yr}$ & $615 / 3377$ & $1.31(1.18-1.45)$ & $0.94(0.87-1.02)$ & $1.04(0.96-1.12)$ \\
\hline White & $483 / 6709$ & $1.43(1.26-1.62)$ & $1.02(0.92-1.13)$ & $1.12(1.02-1.24)$ \\
\hline Black & $165 / 2882$ & $1.21(1.02-1.43)$ & $0.68(0.54-0.85)$ & $0.76(0.63-0.91)$ \\
\hline Mexican & $134 / 3392$ & $1.11(0.95-1.29)$ & $0.78(0.61-0.99)$ & $0.84(0.63-1.12)$ \\
\hline High BMI & $356 / 6011$ & $1.37(1.19-1.59)$ & $1.20(1.01-1.42)$ & $1.39(1.21-1.62)$ \\
\hline Low BMI & $472 / 8094$ & $1.31(1.12-1.51)$ & $0.50(0.40-0.62)$ & $0.80(0.64-1.00)$ \\
\hline$\geq 3$ yr follow up & $408 / 11346$ & $1.32(1.15-1.52)$ & $1.01(0.90-1.14)$ & $1.10(1.00-1.22)$ \\
\hline \multicolumn{5}{|c|}{ Interaction term p-values } \\
\hline *female & & 0.81 & 0.24 & 0.17 \\
\hline *age & & 0.93 & 0.28 & 0.58 \\
\hline$*$ white & &,+ 0.005 & 0.11 & 0.052 \\
\hline *black & & 0.21 &,$-<0.001$ &,$-<0.001$ \\
\hline$*$ mexican & & 0.07 & 0.16 & 0.16 \\
\hline$* \mathrm{bmi}$ & & 0.36 &,$+<0.001$ &,$+<0.001$ \\
\hline
\end{tabular}

Cox proportional hazard modeling for mortality hazard ratio per unit of ABSI, BMI or WC z score (standard deviation). Ranges in parentheses are $95 \%$ confidence intervals. The sign of significant interaction terms is given. High BMI is defined as exceeding the age- and sex-specific population mean.

doi:10.1371/journal.pone.0039504.t003

mortality risk given both values is the product of that due to each separately (Figure 2a, b). Lowest risk is found at below-average ABSI and near average BMI (lower middle of plot), while risk elevations of above 100\% are found at high ABSI and BMI either below or above average (upper left and upper right corners of plot).

We found that our main results continued to hold when the original variables, rather than their $\mathrm{z}$ scores, were used as mortality predictors. Log mortality risk increased steadily with ABSI, while decreasing for increasing BMI and WC up to values around the population median (Figure 4). The association of ABSI with mortality hazard remained after adjusting for other known risk factors (Table 5).

Table 4. Mortality by quintile.

\begin{tabular}{llll}
\hline \multicolumn{5}{c}{ Hazard ratio } & & \\
\cline { 2 - 4 } \begin{tabular}{l} 
Quintile \\
\cline { 2 - 4 }
\end{tabular} & ABSI & BMI & WC \\
\hline 1 (lowest) & $0.97(0.69-1.37)$ & $1.88(1.44-2.45)$ & $1.51(1.12-2.03)$ \\
2 & $0.93(0.64-1.35)$ & $1.23(0.89-1.69)$ & $1.31(0.92-1.87)$ \\
3 (reference) & 1 & 1 & 1 \\
4 & $1.46(1.08-1.99)$ & $1.37(0.95-1.97)$ & $1.30(0.95-1.77)$ \\
5 (highest) & $1.93(1.39-2.68)$ & $1.71(1.22-2.39)$ & $1.72(1.28-2.32)$ \\
\hline $\begin{array}{l}\text { Cox proportional hazard modeling for mortality risk with ABSI, BMI or WC z } \\
\text { score quintiles taken as the predictors. Hazard ratios are relative to the middle } \\
\text { quintile in each case. Ranges in parentheses are 95\% confidence intervals. } \\
\text { The between-quintile cut points are }-0.868,-0.272,+0.229,+0.798 \text { for ABSI; } \\
-0.759,-0.337,+0.113,+0.746 \text { for BMl; and }-0.826,-0.346,+0.120,+0.775\end{array}$ \\
$\begin{array}{l}\text { for WC. } \\
\text { doi:10.1371/journal.pone.0039504.t004 }\end{array}$
\end{tabular}

\section{Discussion}

The newly developed and applied ABSI is based on WC, weight and height, where high ABSI indicates that WC is higher than expected for a given height and weight and corresponds to a more central concentration of body volume. Applying ABSI along with $\mathrm{BMI}$ as a predictor variable separates the influence of the component of body shape measured by WC from that of body size. Our finding that higher ABSI predicts mortality hazard is thus quite analogous to the outcome of analyses which have adjusted WC for BMI without invoking ABSI. Thus, an analysis of mortality outcomes in an elderly ( $\geq 65 \mathrm{yr}$ ) USA cohort found that including both BMI and WC as continuous variables in a Cox proportional hazard model for mortality results in a direct correlation between WC and mortality and an inverse correlation between BMI and mortality [12]. In a large multination European cohort, stratifying by BMI category transformed the curve of mortality risk as a function of WC from U-shaped to more linear, similar to our curve of mortality risk as a function of ABSI quantile [14]. Our work also follows on findings that dividing WC by height increases its ability to predict cardiometabolic risk factors [32-35]. Some conceptual advantages of introducing ABSI are that it accounts for the sublinear increase of WC with BMI (i.e. $\mathrm{WC} \propto \mathrm{BMI}^{2 / 3}$ ) along with the nonlinear association of WC with height, and that using it instead of WC avoids inflation of regression uncertainty associated with the near collinearity of WC and BMI

In the USA population as sampled in NHANES, ABSI predicted mortality risk across age, sex, and weight, although the ethnic difference found suggests that analysis of more cohorts is needed to delineate the limits of ABSI's utility. A logical next step would be to investigate the association of ABSI with longer-term 

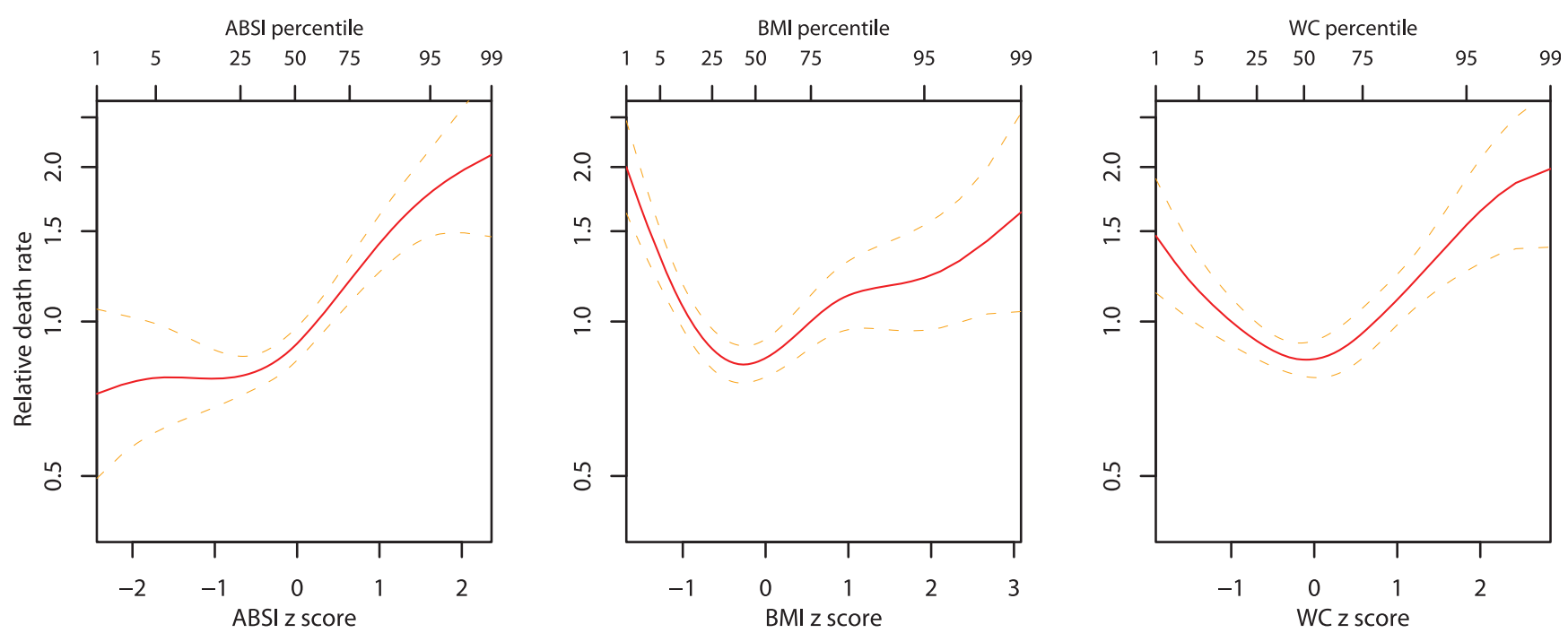

Figure 2. Mortality hazard by ABSI, BMI, and WC $z$ score relative to age and sex specific normals. Estimates are from proportional hazard modeling where log mortality hazard is a smoothing-spline function in ABSI, BMI, or WC. Dashed curves show $95 \%$ confidence intervals. Corresponding population percentiles are given in the top axis; the range shown is the 1st through 99th percentiles. The vertical axis is logarithmic. doi:10.1371/journal.pone.0039504.g002

mortality risk, as well as its ability to predict morbidity and impaired quality of life.

What aspect of human physiology measured by ABSI accounts for its association with death rate? At a given height and weight, high ABSI may correspond to a greater fraction of visceral (abdominal) fat compared to peripheral tissue. As mentioned in the Introduction, excess visceral fat has been associated with a variety of potentially adverse metabolic changes. Equally important may

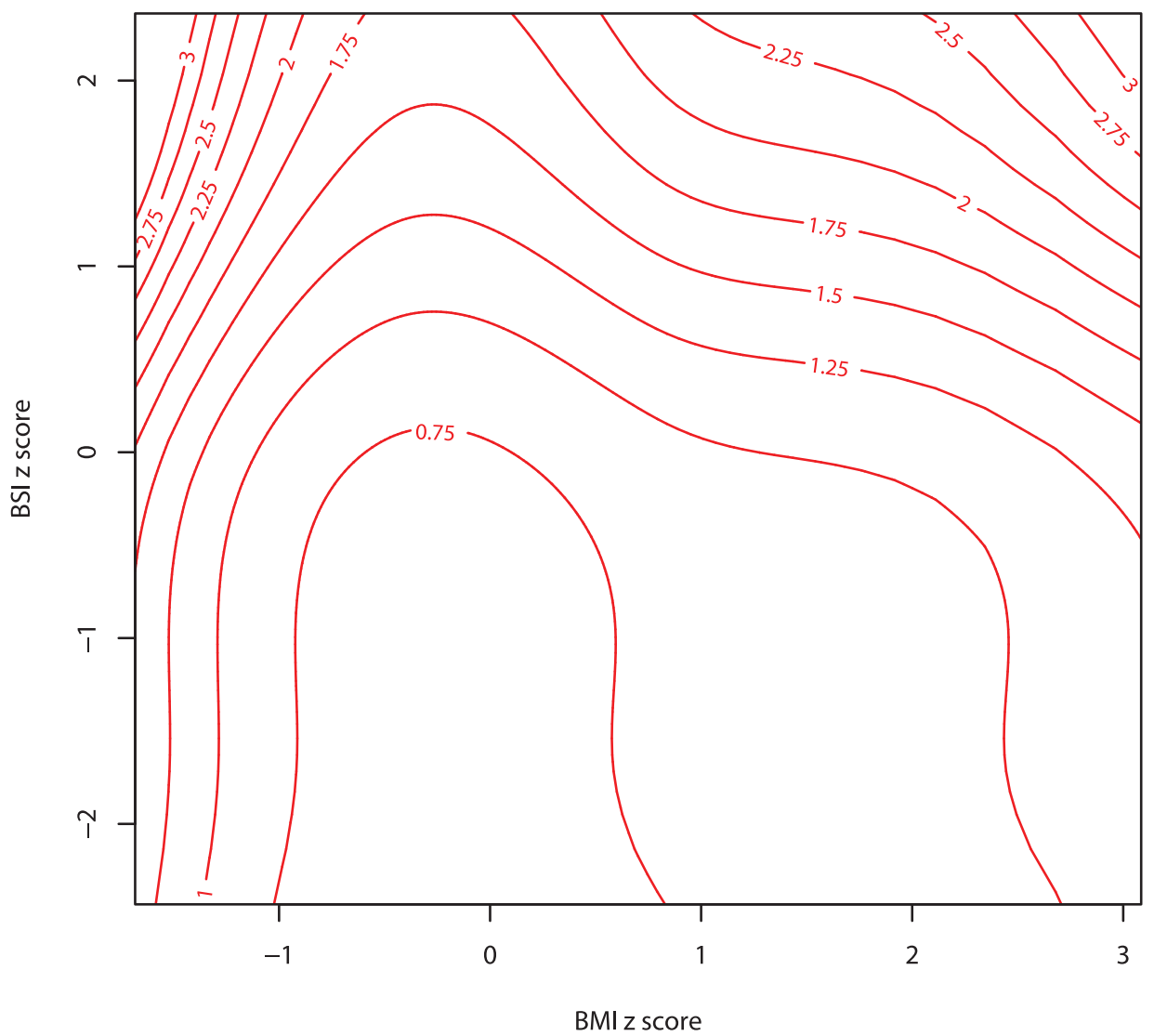

Figure 3. Estimated mortality hazard (relative to the population mean) by combination of BMI and ABSI $z$ Score. The ranges of BMI and ABSI shown correspond to the 1st through 99th percentiles. The contour interval is 0.25 .

doi:10.1371/journal.pone.0039504.g003 

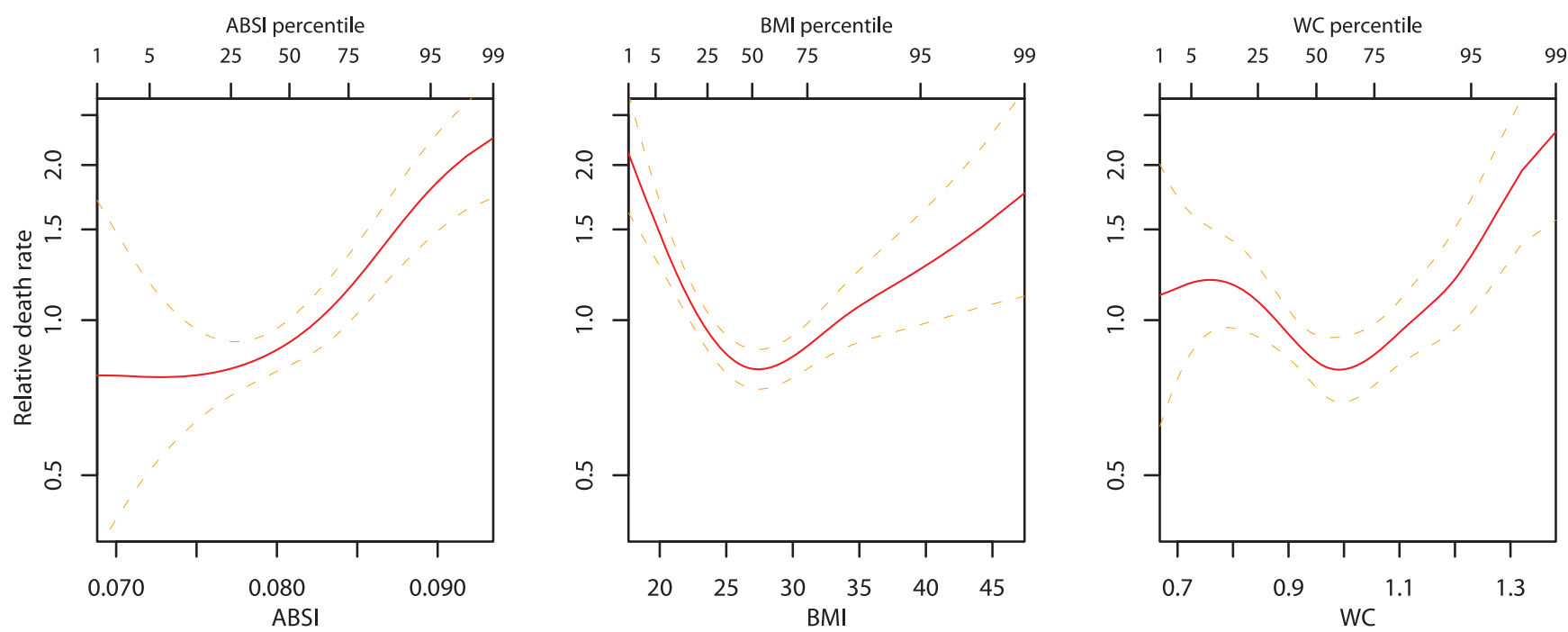

Figure 4. Mortality hazard by ABSI, BMI, and WC. Estimates are from proportional hazard modeling where log mortality hazard is a smoothingspline function in ABSI, BMI, or WC. Dashed curves show $95 \%$ confidence intervals. Corresponding population percentiles are given in the top axis; the range shown is the 1st through 99th percentiles. The vertical axis is logarithmic. This is the same as Figure 2, but with ABSI, BMI, or WC, rather than their $\mathrm{z}$ scores, used as predictors. Units are $\mathrm{m}^{11 / 6} \mathrm{~kg}^{-2 / 3}$ for $\mathrm{ABSI}, \mathrm{kg} \mathrm{m}^{-2}$ for BMI, and $\mathrm{m}$ for WC.

doi:10.1371/journal.pone.0039504.g004

be that individuals with high ABSI have a smaller fraction of mass as limb muscle; lean tissue mass and limb circumference have been shown to have strong negative correlations with mortality risk [7,36]. Dual energy X-ray absorptimetry body composition data for most of the NHANES 1999-2004 sample is available [37,38] and could be used to investigate ABSI's associations with muscle, fat and bone fraction by site. For example, we found that ABSI is positively correlated to trunk fat mass as estimated from X-ray scans $(r=+0.45$ between $\mathrm{z}$ score of trunk fat mass adjusted for height and weight and ABSI z score) but negatively correlated with limb lean mass $(r=-0.26)$, consistent with the above hypotheses; by contrast, WC has only weak associations with both trunk fat and limb lean mass after these are adjusted for height and weight $(r=-0.04$ for both), suggesting that it is a less consistent indicator of changes in body shape and composition not reflected in height and weight.

We found that both low and high BMI increased the mortality hazard compared to near-median BMI (U-shaped curve for

Table 5. Body size and shape and mortality hazard.

\begin{tabular}{llll}
\hline \multicolumn{4}{l}{ Hazard ratio per SD increase } \\
\cline { 2 - 4 } & Unadjusted & Restricted & Adjusted \\
\hline ABSI & $1.39(1.23-1.57)$ & $1.44(1.26-1.63)$ & $1.34(1.18-1.51)$ \\
BMI & $1.00(0.88-1.13)$ & $0.99(0.87-1.13)$ & $0.97(0.84-1.12)$ \\
WC & $1.08(0.97-1.21)$ & $1.10(0.97-1.25)$ & $1.06(0.93-1.22)$ \\
\hline
\end{tabular}

Results of Cox proportional hazard modeling for mortality risk with $\mathrm{ABSI}, \mathrm{BMI}$, or WC, as well as sex, taken as linear predictors. Ranges in parentheses are $95 \%$ confidence intervals. The restricted models are unadjusted but included only those people who had all the measurements required for the adjusted model. The adjusted models included as additional predictors ethnicity, smoking, presence of diabetes, blood pressure, and serum cholesterol.

This is the same as Table 2, but with ABSI, BMI, or WC, rather than their z scores, used as predictors.

$\mathrm{SD}=$ standard deviation. The population standard deviations used here are $0.00535 \mathrm{~m}^{11 / 6} \mathrm{~kg}^{-2 / 3}$ for ABSI, $6.20 \mathrm{~kg} \mathrm{~m}^{-2}$ for BMl, and $0.155 \mathrm{~m}$ for WC.

doi:10.1371/journal.pone.0039504.t005 mortality hazard vs. BMI and WC in Figure 2). In the studied population, the hazard sustained by low BMI quantiles appears to be at least as great than that sustained by corresponding high BMI quantiles, consistent with the nonsignificantly negative linear regression coefficient for mortality hazard on BMI $\mathrm{z}$ score (Table 2). This substantial mortality hazard for low BMI held even though few (292/14105 or 2.1\%) of the study population were in the WHO 'underweight' category of BMI $<18.5 \mathrm{~kg} \mathrm{~m}^{-2}$. The lowest mortality hazard was for the middle quintile of both BMI and WC, although the population median was well in the WHO 'overweight' or 'pre-obese' category [39]: the 40th-60th percentile range for the sample was $25.6-28.4 \mathrm{~kg} \mathrm{~m}^{-2}$, with the exact cutoffs for the middle quintile of BMI z score varying by age and sex (Figure 1c; cf. Figure 4b). Similarly, the 40th-60th percentile range of population WC was $94-101 \mathrm{~cm}$ for men and 88-97 cm for women, above most suggested cut-off points for higher mortality hazard [18]. These results add to many previous studies that show high population mortality hazard even in developed countries from underweight compared to overweight, particularly among the elderly and chronically ill [15,40-45], supporting a rethinking of BMI-based obesity thresholds [46]. However, since high ABSI appears to identify increased mortality risk independent of BMI, it could complement either low or high BMI in risk assessment, as illustrated in Figure 3.

In addition to WC, inverse hip circumference, or waist to hip ratio, have been suggested as alternative measures of body shape that predict mortality better than BMI $[18,47]$. It is theorized that gluteofemoral fat may benefit health by removing free fatty acids from the bloodstream [48]. Different studies have reached a range of conclusions about whether WC $[14,16]$ or waist to hip ratio $[13,17]$ is a better predictor of mortality; a meta-analysis of British studies found them to be equally good predictors [49]. A recent prospective analysis from Mauritius found that higher WC and lower hip circumference both correlated with greater mortality risk, while BMI did not correlate with mortality risk [50]. An analysis of an earlier NHANES cohort (NHANES III, examined 1988-1994) found that neither low nor high waist to hip ratio significantly affected mortality hazard compared to an intermediate reference level, where levels were defined by analogy with 
WHO obesity categories [41]. That study also found that waist to hip ratio, despite its nondimensional form, was significantly correlated to BMI $(r \approx 0.4-0.5)$, and we may hypothesize that adjusting hip circumferences or waist to hip ratios for height and weight, as done here for $\mathrm{WC}$, would make them more useful as predictors of mortality hazard. The significance of hip circumference or waist to hip ratio cannot be evaluated with NHANES 1999-2004 data because hip circumference was not measured, though it is possible that adjusting WC for height and weight may indirectly provide similar information to waist to hip ratio - i.e. a wider waist for given height and weight may imply narrower hips, and vice versa. It would be of interest to compare the waist to hip ratio's performance with that of ABSI in suitable data sets, and it may well be that ABSI can be improved as an indicator of hazardous body shape by including hip circumference in addition to height, weight and WC (which is why ABSI for now bears the indefinite article).

This prospective study does not directly address whether interventions aimed at reducing ABSI would reduce mortality risk, independent of weight change, for which large randomized controlled trials would be necessary. If ABSI does reflect malleable body shape and composition attributes, however, we may speculate that the effectiveness of weight loss interventions in improving health outcomes would be affected by how they impact WC relative to weight, since ABSI varies with the ratio $\frac{\mathrm{WC}}{\mathrm{BMI}^{2 / 3}}$. Lifestyle change that reduces ABSI, such as an exercise program that builds skeletal muscle, may yield health benefits independent of the amount of weight loss; indeed, exercise has been shown to have beneficial health impacts for obese individuals, including reductions in WC (and hence ABSI), even when weight loss does not occur [51]. Weight loss programs including either low-calorie diets or exercise can also reduce $\mathrm{WC}$, along with $\mathrm{BMI}$, enough to reduce ABSI [52,53]. As other possible applications, the strong association of ABSI with mortality may be of interest to actuaries [54], and may be used as a selection criterion for enrollment in clinical trials desired to have higher power to detect mortality outcome differences with given sample size. Note that since ABSI varies over a small range (population standard deviation is of order $5 \%$, Figure $1 \mathrm{~b}$ ), it is sensitive to the accuracy of the biometric

\section{References}

1. WHO (2009) Global health risks: Mortality and burden of disease attributable to selected major risks. Technical report, World Health Organization. URL http://www.who.int/entity/healthinfo/global_burden_disease/ GlobalHealthRisks_report_full.pdf.

2. NHLBI (1998) Clinical guidelines on the identification, evaluation, and treatment of overweight and obesity in adults: The evidence report. Technical Report 98-4083, National Heart Lung and Blood Institute, National Institutes of Health. URL http://www.nhlbi.nih.gov/guidelines/obesity/ob_gdlns.pdf.

3. Nevill AM, Stewart AD, Olds T, Holder R (2006) Relationship between adiposity and body size reveals limitations of BMI. American Journal of Physical Anthropology 129: 151-156.

4. Heymsfield SB, Scherzer R, Pietrobelli A, Lewis CE, Grunfeld C (2009) Body mass index as a phenotypic expression of adiposity: quantitative contribution of muscularity in a population-based sample. International Journal of Obesity 33: 1363-1373.

5. Gómez-Ambrosi J, Silva C, Galofré JC, Escalada J, Santos S, et al. (2012) Body mass index classification misses subjects with increased cardiometabolic risk factors related to elevated adiposity. International Journal of Obesity 36: 286294.

6. Bray GA, Smith SR, de Jonge L, Xie H, Rood J, et al. (2012) Effect of dietary protein content on weight gain, energy expenditure, and body composition during overeating. Journal of the American Medical Association 307: 47-55.

7. Bigaard J, Frederiksen K, Tjonneland A, Thomsen BL, Overvad K, et al. (2004) Body fat and fat-free mass and all-cause mortality. Obesity 12: 1042-1049.

8. Ruhl CE, Everhart JE (2010) Trunk fat is associated with increased serum levels of alanine aminotransferase in the United States. Gastroenterology 138: 1346 1356 . measurements on which it is based. In particular, WC should be measured according to the NHANES protocol [22] in order to meaningfully compare ABSI with the population normals given here, even though in general the association of WC with health outcomes seems independent of the specified measurement protocol [55].

In summary, body shape, as measured by ABSI, appears to be a substantial risk factor for premature mortality in the general population derivable from basic clinical measurements. ABSI expresses the excess risk from high $\mathrm{WC}$ in a convenient form that is complementary to BMI and to other known risk factors.

\section{Supporting Information}

Table S1 This table contains the mean and standard deviation of ABSI by age and sex for NHANES 1999-2004, based on all individuals with available data (except pregnant women) and weighted to represent the larger USA population which NHANES was intended to sample. The smoothed means and standard deviations shown were used to generate ABSI z scores. The table contains 11 space-separated columns in plain text. The layout is: Column 1: Age (85 includes those older than 85). Column 2: Number of males with available data at this age. Column 3: ABSI mean for males at the given age. Column 4: ABSI standard deviation for males at the given age. Column 5: Smoothed ABSI mean for males at the given age. Column 6: Smoothed ABSI standard deviation for males at the given age. Columns 7-11: Same as columns 2-6, but for females. (TXT)

\section{Acknowledgments}

We thank Steven Heymsfield, Michael Kleerekoper, James Levine, and Tom Rifai for valuable discussions and encouragement.

\section{Author Contributions}

Conceived and designed the experiments: NYK JCK. Performed the experiments: NYK JCK. Analyzed the data: NYK JCK. Contributed reagents/materials/analysis tools: NYK JCK. Wrote the paper: NYK JCK.

9. Kang SM, Yoon JW, Ahn HY, Kim SY, Lee KH, et al. (2011) Android fat depot is more closely associated with metabolic syndrome than abdominal visceral fat in elderly people. PLoS ONE 6: e27694.

10. Katzmarzyk PT, Barreira TV, Harrington DM, Staiano AE, Heymsfield SB, et al. (2012) Relationship between abdominal fat and bone mineral density in white and African American adults. Bone 50: 576-579.

11. Lumeng CN, Saltiel AR (2011) Inflammatory links between obesity and metabolic disease. Journal of Clinical Investigation 121: 2111-2117.

12. Janssen I, Katzmarzyk PT, Ross R (2005) Body mass index is inversely related to mortality in older people after adjustment for waist circumference. Journal of the American Geriatrics Society 53: 2112-2118.

13. Simpson JA, MacInnis RJ, Peeters A, Hopper JL, Giles GG, et al. (2007) A comparison of adiposity measures as predictors of all-cause mortality: the Melbourne Collaborative Cohort Study. Obesity 15: 994-1003.

14. Pischon T, Boeing H, Hoffmann K, Bergmann M, Schulze M, et al. (2008) General and abdominal adiposity and risk of death in Europe. New England Journal of Medicine 359: 2105-2120.

15. Kuk JL, Ardern CI (2009) Influence of age on the association between various measures of obesity and all-cause mortality. Journal of the American Geriatrics Society 57: 2077-2084

16. Seidell JC (2010) Waist circumference and waist/hip ratio in relation to all-cause mortality, cancer and sleep apnea. European Journal of Clinical Nutrition 64: 35-41.

17. Petursson H, Sigurdsson JA, Bengtsson C, Nilsen TIL, Getz L (2011) Body configuration as a predictor of mortality: comparison of five anthropometric measures in a 12 year follow-up of the Norwegian HUNT 2 study. PLoS ONE 6: e26621. 
18. WHO (2011) Waist Circumference and Waist-Hip Ratio: Report of a WHO Expert Consultation, Geneva, 8-11 December 2008. Technical report, World Health Organization.

19. Moore SC (2009) Waist versus weight - which matters more for mortality? American Journal of Clinical Nutrition 89: 1003-1004.

20. Klein S, Allison DB, Heymsfield SB, Kelley DE, Leibel RL, et al. (2007) Waist circumference and cardiometabolic risk: a consensus statement from Shaping America's Health. Diabetes Care 30: 1647-1652.

21. NCHS (2011). National Health and Nutrition Examination Survey data. URL http://www.cdc.gov/nchs/nhanes/nhanes_questionnaires.htm.

22. NHANES (2002) National Health and Nutrition Examination Survey anthropometry procedures manual. Technical report, National Center for Health Statistics. URL http://www.cdc.gov/nchs/data/nhanes/nhanes_01_ 02/body_measures_year_3.pdf.

23. NCHS (2006) Analytic and Reporting Guidelines: The National Health and Nutrition Examination Survey (NHANES). Technical report, National Center for Health Statistics, Centers for Disease Control and Prevention, Hyattsville, Maryland. URL http://www.cdc.gov/nchs/data/nhanes/nhanes_03_04/ nhanes_analytic_guidelines_dec_2005.pdf.

24. Burton R (2010) Waist circumference as an indicator of adiposity and the relevance of body height. Medical Hypotheses 75: 115-119.

25. MacKay N (2010) Scaling of human body mass with height: The body mass index revisited. Journal of Biomechanics 43: 764-766.

26. Heymsfield SB, Heo M, Pietrobelli A (2011) Are adult body circumferences associated with height? Relevance to normative ranges and circumferential indexes. American Journal of Clinical Nutrition 93: 302-307.

27. Hansen PG (1998) Rank-deficient and Discrete Ill-posed Problems: Numerical Apects of Linear Inversion. SIAM.

28. Kom EL, Graubard BI, Midthune D (1997) Time-to-event analysis of longitudinal follow-up of a survey: choice of the time-scale. American Journal of Epidemiology 145: 72-80.

29. Therneau TM, Grambsch PM (2000) Modeling survival data: extending the Cox model. New York: Springer-Verlag.

30. Keele L (2010) Proportionally difficult: testing for nonproportional hazards in Cox models. Political Analysis 18: 189-205.

31. Lumley T (2004) Analysis of complex survey samples. Journal of Statistical Software 9: 8

32. Hsieh SD, Yoshinaga H, Muto T (2003) Waist-to-height ratio, a simple and practical index for assessing central fat distribution and metabolic risk in Japanese men and women. International Journal of Obesity 27: 610-616.

33. Browning LM, Hsieh SD, Ashwell M (2010) A systematic review of waist-toheight ratio as a screening tool for the prediction of cardiovascular disease and diabetes: 0.5 could be a suitable global boundary value. Nutrition Research Reviews 23: 247-269.

34. Ashwell M, Gunn P, Gibson S (2012) Waist-to-height ratio is a better screening tool than waist circumference and BMI for adult cardiometabolic risk factors: systematic review and meta-analysis. Obesity Reviews 13: 275-286.

35. Li WC, Chen IC, Chang YC, Loke SS, Wang SH, et al. (2011) Waist-to-height ratio, waist circumference, and body mass index as indices of cardiometabolic risk among 36,642 Taiwanese adults. European Journal of Nutrition.

36. Heitmann BL, Frederiksen P (2009) Thigh circumference and risk of heart disease and premature death: prospective cohort study. British Medical Journal 339: b3292.

37. Kelly TL, Wilson KE, Heymsfield SB (2009) Dual energy x-ray absorptiometry body composition reference values from NHANES. PLoS ONE 4: e7038.
38. Sun Q van Dam RM, Spiegelman D, Heymsfield SB, Willett WC, et al. (2010) Comparison of dualenergy x-ray absorptiometric and anthropometric measures of adiposity in relation to adiposityrelated biologic factors. American Journal of Epidemiology 172: 1442-1454.

39. WHO (2000) Obesity: preventing and managing the global epidemic. Technical Report WHO Obesity Technical Report Series No. 894, World Health Organization.

40. Allison DB, Gallagher D, Heo M, Pi-Sunyer FX, Heymsfield SB (1997) Body mass index and allcause mortality among people age 70 and over: the Longitudinal Study of Aging. International Journal of Obesity 21: 424-431.

41. Flegal KM, Graubard BI (2009) Estimates of excess deaths associated with body mass index and other anthropometric variables. American Journal of Clinical Nutrition 89: 1213-1219.

42. Gulsvik AK, Thelle DS, Mowé M, Wyller TB (2009) Increased mortality in the slim elderly: a 42 years follow-up study in a general population. European Journal of Epidemiology 24: 683-690.

43. Atlantis E, Browning C, Kendig $\mathrm{H}$ (2010) Body mass index and unintentional weight change associated with all-cause mortality in older Australians: the Melbourne Longitudinal Studies on Healthy Ageing (MELSHA). Age and Ageing 39: 559-565.

44. Cepeda-Valery B, Pressman GS, Figueredo VM, Romero-Corral A (2011) Impact of obesity on total and cardiovascular mortality - fat or fiction? Nature Reviews Cardiology 8: 233-237.

45. Nilsson G, Hedberg P, Öhrvik J (2011) Survival of the fattest: unexpected findings about hyperglycaemia and obesity in a population based study of 75year-olds. BMJ Open 1: e000012.

46. Campos P, Saguy A, Ernsberger P, Oliver E, Gaesse G (2006) The epidemiology of overweight and obesity: public health crisis or moral panic? International Journal of Epidemiology 35: 55-60.

47. Heitmann BL, Lissner L (2011) Hip Hip Hurrah! Hip size inversely related to heart disease and total mortality. Obesity Reviews 12: 478-481.

48. Manolopoulos KN, Karpe F, Frayn KN (2010) Gluteofemoral body fat as a determinant of metabolic health. International Journal of Obesity 34: 949-959.

49. Czernichow S, Kengne AP, Stamatakis E, Hamer M, Batty GD (2011) Body mass index, waist circumference and waist-hip ratio: which is the better discriminator of cardiovascular disease mortality risk? Evidence rom an individual-participant meta-analysis of 82864 participants from nine cohort studies. Obesity Reviews 12: 680-687.

50. Cameron AJ, Magliano DJ, Shaw JE, Zimmet PZ, Carstensen B, et al. (2012) The influence of hip circumference on the relationship between abdominal obesity and mortality. International Journal of Epidemiology.

51. King NA, Hopkins M, Caudwell P, Stubbs RJ, Blundell JE (2009) Beneficial effects of exercise: shifting the focus from body weight to other markers of health. British Journal of Sports Medicine 43: 924-927.

52. Han TS, Richmond P, Avenell A, Lean MEJ (1997) Waist circumference reduction and cardiovascular benefits during weight loss in women. International Journal of Obesity 21: 127-134.

53. Ross R, Dagnone D, Jones PJ, Smith H, Paddags A, et al. (2000) Reduction in obesity and related comorbid conditions after diet-induced weight loss or exercise-induced weight loss in men. Annals of Internal Medicine 133: 92-103.

54. Kuh D, Hardy R, Hotopf M, Lawlor DA, Maughan B, et al. (2009) A review of life time risk factors for mortality. British Actuarial Journal 15: 17-64.

55. Ross R, Berentzen T, Bradshaw AJ, Janssen I, Kahn HS, et al. (2008) Does the relationship between waist circumference, morbidity and mortality depend on measurement protocol for waist circumference? Obesity Reviews 9: 312-325. 